\title{
Relatos metodológicos: destaques do estudo "Brazilian Model of Library and Information Studies in the bachelor's level"
}

\section{Methodological reports: highlights of the study "Brazilian Model of Library and Information Studies in the bachelor's level"}

\author{
Plácida L. V. Amorim da Costa Santos ${ }^{1}$, Chaim Zins ${ }^{2}$ \\ ${ }^{1}$ Universidade Estadual Paulista - UNESP/Marília, Marília, SP, Brasil \\ ${ }^{2}$ Knowledge Mapping Research, Jerusalem, Israel
}

Autor para correspondência/Mail to: Plácida L.V. Amorim da Costa Santos (placida@marilia.unesp.br)

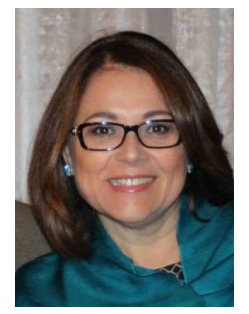

Plácida L. V. Amorim da Costa Santos Bacharel em Biblioteconomia pela Universidade Estadual Paulista (UNESP), Mestre em Ciência da Informação pela Pontificia Universidade Católica de Campinas (PUC-Campinas), Doutorado em Letras pela Universidade de São Paulo (USP) e Livre-Docente em Catalogação pela Universidade Estadual Paulista (UNESP). Docente no Programa de Pós-Graduação em Ciência da Informação - PPGCI/UNESP-Marília

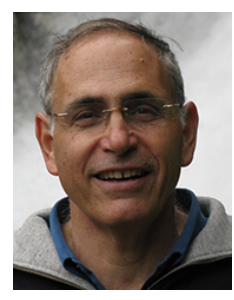

Chaim Zins Doctor of Philosophy. Cientista da informação, Estudioso, educador e artista conceitual. Especialista em mapeamento do conhecimento [http://www.success.co.il]. Principais projetos de investigação: 10 Pillars of Knowledge; Knowledge Map of Information Science; Knowledge Map of Judaism (Hebrew); and, the Critical Delphi research methodology.

Notas/Notes: Os questionamentos desta entrevista foram elaborados com base no artigo "Brazilian Model of Library and Information Studies in the Bachelor's level" publicado na revista Informação \& Sociedade: estudos, v. 25, n. 1, de 2015. Disponível em: http://www.ies.ufpb.br/ojs/index.php/ies/article/view/26761/14536.

Copyright (c) 2016 Santos \& Zins. Todo o conteúdo da Revista (incluindo-se instruções, política editorial e modelos) está sob uma licença Creative Commons Atribuição-NãoComercial-Compartilhalgual 3.0 Não Adaptada. Ao serem publicados por esta Revista, os artigos são de livre uso em ambientes educacionais, de pesquisa e não comerciais, com atribuição de autoria obrigatória. Mais informações em http://ojs.c3sl.ufpr.br/ojs2/index.php/atoz/about/submissions\#copyrightNotice.

\begin{abstract}
Resumo
Os Doutores Plácida Santos e Chaim Zins detalham as principais opções metodológicas da exaustiva investigação voltada à formação de profissionais em Ciência da Informação no Brasil. Comentam sobre a continuidade dos estudos na temática e as contribuições que os atuais resultados podem - potencialmente - trazer aos programas de graduação na área.
\end{abstract}

Palavras-chave: Estudo Delphi; Teoria fundamentada em dados; Formação profissional

\begin{abstract}
Doctors Placida Santos and Chaim Zins detail the main methodological options of their exhaustive research regarding training professionals in Information Science in Brazil. They emphasize the continuation of studies on the subject and the potential contributions to undergraduate programs in the area.

Keywords: Delphi Study; Grounded Theory; Information profesionals trainning
\end{abstract}

1. Em um processo de investigação utilizando o método Delphi quais são as fases/etapas mais desafiadoras? Por quê?

Um estudo Delphi crítico é, geralmente, realizado em três etapas. Primeiro, o pesquisador faz as perguntas. Em seguida, ele/ela esclarece as posições dos painelistas, confrontando-as com as diversas respostas. Finalmente, ele/ela resume as conclusões. Se necessário, existe uma quarta etapa na qual aos membros do painel é solicitada uma nova verificação e reavaliação de suas posições. A fase mais difícil é a primeira em que o pesquisador define as principais questões e âmbito do estudo. A fase mais importante é a última (terceira fase) quando o pesquisador resume as conclusões.

O método Delphi é empregado em vários campos, como estudos econômicos, evolução de mercados, desenvolvimento socioeconômico, progresso tecnológico e educação, entre outros. O propósito fundamental é esclarecer aspectos sobre a evolução de uma dada situação, para identificar prioridades ou para apresentar diferentes cenários prospectivos. $\mathrm{O}$ emprego do método procura a efetiva utilização do julgamento intuitivo, com base nas 
opiniões de especialistas, que são refinadas em um processo interativo e repetido algumas vezes até se alcançar o consenso interdisciplinar e correspondente à redução do viés individual e idiossincrasias sobre o assunto abordado, com a finalidade de delinear e realizar previsões.

O método Delphi, em nosso estudo, foi aplicado usando-se questionários para respostas escritas. Um questionário simples, interativo, circulou repetidas vezes pelo painel de especialistas e foi consolidado de modo a associar escalas qualitativas ou quantitativas às questões. Os instrumento de coleta foram submetidos aos especialistas em uma sequência de 3 (três) rodadas.

A cada rodada os pesquisadores contabilizaram as respostas, apresentaram os resultados parciais, normalmente sob a forma de descritores estatísticos simples e solicitaram aos especialistas uma revisão, em anonimato, de suas opiniões à luz da opinião agregada. Cada participante pode fornecer então um novo julgamento, justificando a mudança ou não de opinião. O processo se repetiu até que foi atingido um "estado estacionário".

Desafios encontrados durante o estudo foram referentes a:

Formulação das perguntas: A escolha das perguntas e a sua formulação foram bastante cuidadosas, no sentido de obter o máximo de informação com o menor número de perguntas. O desafio foi elaborar um questionário sem ambiguidades e não enviesado sobre tendências futuras, evitando perguntas que pudessem levar a uma resposta induzida ou que comprometessem a própria legalidade da resposta do entrevistado. A solução foi elaborar um questionário com perguntas curtas, claras e completas, sem ambiguidades nem redundâncias, apresentando uma pequena síntese com as principais informações sobre o assunto e pedindo extrapolações para o futuro;

Seleção do painel: $O$ painel foi composto por 21 participantes. Inicialmente, 58 acadêmicos foram convidados a participar no estudo, 27 concordaram em receber o primeiro questionário, mas apenas 21 responderam, e esses formaram o painel. Os 58 pesquisadores foram selecionados a partir da Plataforma Lattes/CNPq e das associações brasileiras de Ciência da Informação. A representatividade do painel não se deu em relação a uma população, mas em relação ao envolvimento acadêmico e profissional no campo da Ciência da Informação. O desafio foi construir um painel com um número relevante de integrantes, bem preparados e dispostos a se envolver em um estudo dessa natureza;

Construção, aplicação e análise dos questionários: O estudo foi composto por três rodadas sucessivas de questionários estruturados. As discussões entre os membros do painel foram indiretas e anônimas, e foram moderadas pelos pesquisadores. O primeiro questionário foi apresentado ao painel em novembro de 2011. Ele continha 12 questões detalhadas e abertas. O segundo questionário foi apresentado em março de 2012 e continha 23 perguntas. O terceiro questionário foi apresentado em agosto de 2012 e continha 17 questões. O desafio do processo de construção, aplicação e análise dos questionários é o tempo requerido para a realização das rodadas de questionários e o comprometimento dos participantes, pois o feedback é efetuado por meio das sucessivas aplicações de questionários permitindo a troca de informação entre os sujeitos, conduzindo geralmente a uma convergência de consenso. Quando, em algumas vezes não surge o consenso, as respostas ficam polarizadas em duas ou três posições distintas. Na fase de análise dos questionários foi importante identificar que há uma tendência dos participantes em simplificar - de forma notável- suas respostas, pensando em termos de eventos lineares e isolados, em vez de uma visão global da discussão, envolvendo todas as circunstâncias e associações possíveis;

Resultados e conclusões: A análise estatística dos sucessivos resultados obtidos (normalmente de correlação) visou identificar convergências e divergências nas respostas e apresentou como desafio a necessidade de se aclararem as conclusões derivadas da análise dos resultados, de modo a permitir a descrição consistente dos resultados e conclusões do estudo.

\section{Qual foi a contribuição da Grounded Theory para o estudo?}

O estudo Delphi crítico é baseado no pensamento chave das pessoas. O estudo Grounded Theory (ou estudos, no nosso caso) fundamenta as conclusões do estudo Delphi no mundo real (programas acadêmicos e disciplinas em todo o mundo (e no Brasil).

A contribuição da Grounded Theory para a segunda fase do nosso estudo foi muito grande pois, esta é uma metodologia pertinente quando se pretende construir um conjunto sistemático de conceitos, ligados por meio de relações explícitas, capaz de explicar fenômenos e dotado de certa capacidade de previsão, em que dados possam ser integrados, sintetizados e conceitualizados. A utilização da Grounded Theory foi apropriada, pois a pretensão foi a avaliação formativa assistemática das categorias de conteúdos dos modelos para desenvolvimento de programas acadêmicos com o objetivo de identificar processos subjacentes a afirmações já estabelecidas.

Na Grounded Theory a coleta de dados e a análise são feitas simultaneamente. Seu uso permitiu aos pesquisadores analisarem o Modelo Universal ( model $_{1}$ ) em mais de 100 programas de graduação em CI em todo o mundo e o Modelo Brasileiro ( model $_{2}$ ) em 13 programas de graduação no Brasil. A partir dessa análise foram elaboradas estruturas teóricas explanatórias. 
Os dois modelos são compostos por dois modelos complementares: Modelo Universal ( model $_{1.1}$ ) Modelo Brasileiro (model ${ }_{2.1}$ ) em um processo de desenvolvimento sistemático com quatro etapas: a definição dos objetivos acadêmicos, a especificação de conteúdos derivados, a organização do conteúdo no plano estruturado, e a avaliação do programa; e Modelo Universal ( model $\left._{1.2}\right)$ Modelo Brasileiro ( model $_{2.2}$ ) - plano estruturado de conteúdos com 288 categorias.

O model $_{1.2}$ e o model $_{2.2}$ demonstram-se via campos exemplares e de disciplinas levantadas a partir de currículos acadêmicos em todo o mundo e, especificamente, no Brasil. Foram revisados mais de 100 programas em 70 universidades. Nos exemplos nos referimos a 35 universidades de 12 países. No Brasil trabalhamos com 13 programas de 9 universidades; todos eles oferecem um grau de bacharel em estudos de base da Ciência da Informação. Para permitir que os leitores que não falam português acompanhassem os exemplos do Modelo Brasileiro traduzimos a grade curricular dos programas selecionados para a língua inglesa e desenvolvemos o site (http://cienciadainformacao.inf.br) para sua divulgação.

A revisão dos programas acadêmicos tornou-se um bloco de construção metodológica inestimável que desempenhou um papel importante na formação do plano estruturado das categorias de conteúdo ( model $_{1.2} \mathrm{e}$ model $_{2.2}$ ).

Os dois modelos complementares ( model $_{\mathrm{x} .1}$ e model $_{\mathrm{x} .2}$ ) são combinados em model $_{1}$ ou model $_{2}$ integrados, utilizando o ( model $\left._{\mathrm{X} .1}\right)$ - plano estruturado de desenvolvimento - ao se selecionar e ordenar o conteúdo ( model $_{\mathrm{X} .2}$ ).

O estudo foi composto de duas fases metodológicas. A primeira foi um estudo sistemático Delphi crítico com 21 principais estudiosos da Ciência da Informação do Brasil. A segunda fase foi da avaliação formativa assistemática dos model $_{1.2}$ e model $_{2.2}$, que foi baseado em um estudo de Grounded Theory.

\section{Considerando as potencialidades da Grounded Theory e os dois modelos já desenvolvidos, como vocês visua- lizam a continuidade do estudo sobre o ensino em Biblioteconomia e Ciência da Informação?}

A Grounded Theory pode ser considerada como uma técnica de pesquisa ainda em desenvolvimento na Ciência da Informação no Brasil. Trata-se de uma forma rica de se fazer pesquisa e fortemente dependente do pesquisador.

Os modelos apresentados são modelos constitutivos fundamentados em bases teóricas em vez de um espelho empírico de um processo de desenvolvimento que ocorre em uma instituição acadêmica real. Nesse sentido, é importante ressaltar que ambos os modelos precisam ajustes ad hoc, o que permite a continuidade do estudo. Os modelos resultantes do nosso estudo devem ser rigorosamente examinados e implementados no mundo real pelos membros do corpo docente de cada instituição.

Durante o desenvolvimento do estudo uma série de insights surgiram, os quais, mesmo não ligados diretamente à questão de pesquisa, a tangenciam. O estudo pode ser continuado a partir de questões ligadas à análise de situações locais no ensino de graduação em Ciência da Informação, no que diz respeito a concepção e a operacionalização de programas acadêmicos na Ciência da Informação, considerando os objetivos acadêmicos, a organização de conteúdos e a avaliação do curso. Os modelos apresentados podem ser alvo de futuras pesquisas sendo úteis no sentido de entender e de direcionar as instituições de ensino a partir de um plano estruturado em categorias para a seleção de conteúdos para a construção de um raciocínio curricular fundamentado em um determinado contexto.

Importante é considerar que um programa acadêmico é fundamentado na concepção do campo e os objetivos acadêmicos específicos que se pretende alcançar. O programa é direcionado para determinados ambientes acadêmicos, vinculados a determinadas condições sócio culturais, direcionados a determinados alunos, destinados a ser ensinado por dado conjunto de pessoal e a utilização métodos de ensino específicos.

Por fim, cabe destacar que o campo da Ciência da Informação está mudando constantemente e assim é com educação em Ciência da Informação. Portanto, os modelos desenvolvidos no estudo devem ser vistos como parte de uma agenda de pesquisa em andamento.

\section{Quais são as principais diferenças entre o modelo brasileiro de ensino de Biblioteconomia e Ciência da Infor- mação e o modelo universal?}

O modelo universal define os motivos de seleção de conteúdo no ensino de Ciência da Informação em todo o mundo, enquanto o modelo brasileiro exemplifica o modelo universal, implementando-o para o Brasil (em nível nacional). Note-se que o modelo brasileiro também é "universal". Ele define os motivos de seleção de conteúdo no ensino da Ciência da Informação no Brasil. Ainda assim, cada universidade tem de ajustar o modelo ao seu ambiente e condições específicas.

$\mathrm{Na}$ análise das respostas dos questionários notamos que a maioria destas, apesar de baseadas no contexto brasileiro, possibilitaram a descoberta de posições essenciais pertinentes para estudos de Biblioteconomia e Ciência da Informação em todo o mundo. Essa percepção resultou na revisão dos objetivos iniciais do estudo, 
adicionando a perspectiva universal. Consequentemente, o estudo foi ampliado e acabou em dois modelos exemplares inter-relacionados e complementares, um modelo universal ( model $\left._{1}\right)$ e um modelo local ( model $_{2}$ ).

O modelo universal define os princípios orientadores para o desenvolvimento dos cursos de graduação aplicáveis no ensino de Biblioteconomia e Ciência da Informação em todo o mundo. O segundo, o modelo brasileiro, demonstra o raciocínio curricular, definindo os princípios orientadores para o desenvolvimento de programas acadêmicos no Brasil.

O estudo na perspectiva universal resultou em um processo sistemático de desenvolvimento do programa e um esquema estruturado (ou plano) de conteúdos composto de 288 categorias de conteúdo. Ambos, o processo sistemático de desenvolvimento e as 288 categorias de conteúdo, foram estudados em sua aplicação no contexto brasileiro.

\section{Na fala dos membros do painel, em relação ao "Knowledge Map of Information Science" foram identificados temas emergentes ou a necessidade de ampliação/modificação do mapa?}

(Chaim Zins) Nos últimos 15 anos tenho estudado os fundamentos teóricos da Ciência da Informação. O estudo culminou em dois grandes projetos de investigação. O primeiro projeto foi realizado nos anos 2000 . Foi um estudo Delphi crítico com 57 principais estudiosos de 16 países e resultou em seis concepções de Ciência da Informação e em um Mapa de conhecimento em 10 partes.

O segundo projeto teve início em 2011 e foi realizado em conjunto com a minha colega, Plácida Santos. Um estudo Delphi crítico com 21 importantes pesquisadores do Brasil e um estudo baseado na Grounded Theory dos programas de Biblioteconomia e Ciência da Informação em nível de graduação. No decorrer do estudo, a professora Plácida e eu analisamos mais de 100 programas em 70 universidades de 12 países. Ao rever os programas acadêmicos nós identificamos sete principais direções. Todas as direções identificadas estão representadas nas escolas que participam do movimento iSchools.

A primeira direção é no sentido tradicional, (veja Librarianship e Records and Archives Management, da Charles Sturt University); a segunda é a informática (veja bacharelado em Science in Informatics, da University of Melbourne); a terceira direção é informática social (veja o programa de graduação em Social Computing, da University College Dublin); a quarta é sistemas de informação (veja, os três programas de graduação oferecidos pela Cornell University, especialmente o programa da College of Engineering in Information Science, Systems, and Technology, que "estuda a concepção e a gestão de sistemas de informação, com ênfase em informações de engenharia de sistemas em grandes contextos de aplicação"); a quinta é das novas mídias (veja Interactive Digital Media, da University of Toronto, e o programa de especialização Game Production and Innovation, da Rutgers University); a sexta direção é a computação (veja Graduate School of Information Science and Technology, da University of Tokyo); e a sétima direção é de gestão (veja Information and Knowledge Management, da Charles Sturt University).

Eu penso em expandir os resultados dos dois projetos por meio de meus conhecimentos sobre as concepções atuais do campo da Ciência da Informação e as minhas conclusões sobre a sua essência. Eu analisarei as definições de campos de conhecimento explorando a questão de saber se a Ciência da Informação é um campo distinto, um campo interdisciplinar, um campo multidisciplinar, ou - como eu reivindico - um nome genérico, que significa diferentes campos que são geralmente divididos em grupos, baseados nas áreas da sociologia e das tecnologias.

\section{Como vocês projetam o impacto prático deste estudo na comunidade de educadores envolvidos com a for- mação de profissionais da informação no Brasil?}

Ao analisar os programas acadêmicos nós identificamos sete principais direções não exclusivas inter-relacionadas. Na verdade, a maioria dos programas que foram revisados incorporam mais de uma direção.

A primeira direção identificada é o sentido tradicional, que está centrada na Biblioteconomia e na Arquivologia. A segunda direção é a informática, que é centrada na busca e uso de informação em todas as áreas do conhecimento. Note-se que o termo "informática"tem diferentes significados. Aqui ele representa a práxis do trabalho de informação. A terceira direção é a informática social, centrada nos aspectos sociais das indústrias da informação. A quarta direção é a dos sistemas de informação, centrada no desenvolvimento e na utilização de sistemas de informação de base tecnológica. A quinta direção é a de novas mídias. Esta quinta direção amplia o âmbito da Ciência da Informação e engloba Web, celulares e aplicativos de novas mídias, tais como: vídeo, jogos, entretenimento e similares. A sexta direção é a da computação, baseada na integração da Ciência da Informação e a Ciência da Computação. A sétima direção é a de gestão, centrada nos estudos sobre a gestão do conhecimento organizacional, englobando a gestão do conhecimento, gestão de negócios e estudos de gestão.

Ao analisar os programas brasileiros não pudemos evitar a impressão de que o ensino da Ciência da Informação no Brasil, ainda está preso ao estágio tradicional, a primeira direção identificada no estudo, e não voltado às 
tendências atuais. Esta impressão é reforçada quando comparado com o conteúdo dos programas acadêmicos em Biblioteconomia e Ciência da Informação em outros países.

Tal constatação revela a necessidade de revisão dos cursos de graduação, ajustando-os às inovadoras abordagens das tecnologias de informação e comunicação. Muitos dos cursos avaliados têm natureza eclética e oferecem conteúdos que se especializam em tecnologias da informação e na gestão da informação, que vão além das estruturas tradicionais de Biblioteconomia, Arquivologia e Museologia. Fato que indica que a Ciência da Informação, no Brasil está aberta ao que acontece no contexto global, apesar de sua estrutura organizacional rígida.

A necessidade de alterar a estrutura organizacional rígida dos cursos de graduação também emerge da análise das respostas do painel de especialistas sobre a estrutura preferida de cursos de graduação na área da Ciência da Informação. O formato selecionado para uma estrutura de ensino em Biblioteconomia e Ciência da Informação é composto de duas partes de até dois anos. Oito estudiosos sugeriram que se deve dedicar os primeiros dois anos nos estudos gerais e concentrar nos últimos dois anos os estudos baseados em especializações. Se esse formato for aplicado, isso mudará o ensino de graduação em Biblioteconomia e Ciência da Informação no Brasil, que atualmente, parece estar bloqueado em molduras rígidas, e o tornará mais flexível com a adoção de abordagens inovadoras e campos atualizados de especialização. 
Como citar esta entrevista (APA):

Santos, P. L. V. A. C. \& Zins, C. (2016). Relatos metodológicos: destaques do estudo "Brazilian Model of Library and Information Studies in the bachelor's level". AtoZ: novas práticas em informação e conhecimento, 5(1), 4 - 9. Recuperado de: http://dx.doi.org/10.5380/ atoz.v5i1.47327 